\title{
The preventive effect of cellulose from Pleurotus eryngii on fatty liver of hyperlipidemic rats.
}

\author{
Jia-Fu Huang ${ }^{1}$, Yi-Xin Ou${ }^{1,2}$, Xiu-Min Li ${ }^{1}$, Zhi-Chao Lin ${ }^{1}$, Bo Leng ${ }^{1}$, Yu Xue ${ }^{1}$, Yi Su', Yu-Tian Pan ${ }^{\text {* }}$ \\ ${ }^{1}$ Engineering Technological Center of Mushroom Industry, Minnan Normal University, Zhangzhou, Fujian 363000, PR \\ China \\ ${ }^{2}$ State Key Laboratory of Microbial Metabolism, and School of Life Sciences \& Biotechnology, Shanghai Jiao Tong \\ University, Shanghai, 200240, PR China
}

\begin{abstract}
Objective: To investigate the preventive effects of Pleurotus eryngii cellulose (PC) on fatty liver rats with hyperlipemia.

Methods: Rats were fed with different doses of the cellulose coupled with high fat diet containing cholestyramine as positive control. After 5 weeks, we detect the serum levels of alanine aminotransferase (ALT), aspartate aminotransferase (AST), total cholesterol (TC) as well as total triglycerides (TG), and the activities of malonaldehyde (MDA), superoxide dismutase (SOD), hepaticlipase (HL), lipoprteinlipase (LPL) and then we observed the pathological changes in the liver.

Results: P. eryngii cellulose could significantly decrease the ALT, AST, TC and TG serum levels $(P<0.01)$, and improve the activity of SOD and HL while significantly inhibit MDA $(P<0.05)$ in a dose dependent manner. In addition, the histopathological test showed that the livers were milk-yellow and bigger in negative group (rats were not treated with cellulose), and there was no fatty generation or vacuoles in the livers in administration group.

Conclusion: The preventive effect of the cellulose is similar to the cholestyramine $(\mathbf{P}<0.05)$. All results indicated that the cellulose of Pleurotus eryngii has the potential effects on preventing the fatty liver in rats with hyperlipemia.
\end{abstract}

Keywords: Pleurotus eryngii, Cellulose, Hyperlipidemia fatty liver.

Accepted on March 21, 2018

\section{Introduction}

As the development of economy and the changes of living habits and diet structure, the incidence of fatty liver increases rapidly. Fatty liver includes alcoholic and non-alcoholic fatty liver. The later could be divided into obesity fatty liver, hyperlipemia fatty liver, diabetic fatty liver, nutritional disorder fatty liver, drug-induced fatty liver and so on according to their causes [1].

Non-alcoholic fatty liver was not the benign or stationary lesion, which could change into irreversible liver damage in a short time, and then develop into fatty hepatitis, fatty hepatic fibrosis, and fatty cirrhosis [2]. Therefore, it is important to prevent and treat this type fatty liver.

Pleurotus eryngii Que1, also known as the king oyster mushroom, was belonged to mycota, basdiomycotina, hymenomycetes, agarcales, pleurotaceae, agaricochaete [3]. Pleurotus eryngii was with hypertrophic, crisp and tender fleshy, and smelt like abalone and almond. There were abundant proteins, fibers, vitamins and minerals but low fat in Pleurotus eryngii, which were beneficial for human health.
Each $100 \mathrm{~g}$ of Pleurotus eryngii contained $16.2 \mathrm{~g}$ of proteins, $3.8 \mathrm{~g}$ of fat, $64.7 \mathrm{~g}$ of total sugar, $1283 \mathrm{~J}$ of energy, $6.6 \mathrm{~g}$ of cellulose, $0.3 \mathrm{~g}$ of ash, $262.7 \mathrm{mg}$ of potassium, $260 \mathrm{mg}$ of $\mathrm{K}$, $26 \mathrm{mg}$ of calcium, $3.2 \mathrm{mg}$ of $\mathrm{Fe}, 25.3 \mathrm{mg}$ of $\mathrm{Na}$, and vitamin B1, vitamin B2 [4].

Pleurotus eryngii could inhibit the hydrolysis methyl umbelliferone oleate and the emulsification of triolein, and decrease the absorbance of fat, and then decrease the triglyceride (TG) and total cholesterol (TC) levels in serum, reduce the damage of atherosclerosis, regulate the activity of $\mathrm{T}$ helper, improve the generation of cytokines, decrease the invasion of inflammatory cells and mast cell, and release allergic dermatitis [5-8].

Additionally, Pleurotus eryngii could inhibit the growth of helicobacter pylori, Escherichia coli (E. coli), staphylococcus aureus and listeria strains, prevent from virus infection including H1N1, HSV-2 and so on [9-11].

Dietary fiber was partly or totally fermentation in colon, including polysaccharide, oligosaccharide, lignin and plant material, confirmed by Association of Official Analytical 
Chemists (AOAC) in 2001 [12]. It could adsorb fat and excrete outside to decrease the absorption of fat, which was helpful for treating obesity and relative disease [13].

Our study focused on the preventive effect of Pleurotus eryngii cellulose on fatty liver rats with hyperlipemia, in order to provide treatment obesity and healthy diet with evidences.

\section{Materials and Methods}

\section{Materials}

Due to the estrogen might be influence the results, so Sprague Dawley (SD) male rats, SPF, weight $100 \mathrm{~g} \pm 20 \mathrm{~g}$, were obtained from Slac Laboratory Animal Co., Ltd. (SCXK (Hu) 2012-0002, Shanghai, China). All rats were raised in the room at $22 \pm 2{ }^{\circ} \mathrm{C}$ and with freely feeding and drinking for $7 \mathrm{~d}$ to adapt to the environment.

High fat diet $(78.8 \%$ basic diet, $1 \%$ cholesterol, $10 \%$ yolk powder, $10 \%$ lard, $0.2 \%$ bile salt) and basic diet were obtained from Slac Laboratory. PC was obtained from the Engineering Technological Center of Mushroom Industry (Minnan Normal University) and prepared with $0.2 \%$ sodium carboxymethylcellulose. Cholestyramine was obtained from Lifecare Limited (Nanjing, China). ALT, AST, TC and TG detection kits were obtained from Mindary Medical International Limited (Shenzhen, China). SOD and MDA detecting kits were obtained from Nanjing Jiancheng Bioengineering Institute (Nanjing, China).

Automatic biochemistry analyzer (BS-220, Mindary); Histotome (5062, Syil Limited, Ningbo, China); embedding machine (EG1150, Leica Biosystems, Beijing, China); dehydrating machine (TP1020, Leica); ultraviolet spectrophotometer (UV-1100, Mapada Instruments Co., Ltd, Shanghai, China); homogenizer (PRO200, Pro Scientific Inc. Shanghai, China); $-86^{\circ} \mathrm{C}$ refrigerator (Forma $-86^{\circ} \mathrm{C}$, Thermo Fisher Scientific Inc. Shanghai, China); centrifuge (5810, Eppendorf Limited, Shanghai, China); microscopy (BX51, Olympus Imaging China); analytical balance (AUY120, Shimadzu, Hangzhou, China).

\section{Methods}

Groups and drug: 48 SD male rats were divided into 6 groups randomly, including control group, model group (MG), cholestyramin group (CG), Pleurotus eryngii cellulose hightdose group (PCHG), Pleurotus eryngii cellulose middle-dose group (PCMG) and Pleurotus eryngii cellulose low-dose group (PCLG). The rats were fed with basic diet and given with $0.2 \%$ sodium carboxymethylcellulose according to $10 \mathrm{~mL} / \mathrm{kg}$ in control group. The rats in MG were fed with high fat diet and given with $0.2 \%$ sodium carboxymethylcellulose according to $10 \mathrm{~mL} / \mathrm{kg}$. The rats in CG $\left(1 \mathrm{~g} \cdot \mathrm{Kg}^{-1} \cdot \mathrm{d}^{-1}\right)$, PCHG $\left(1 \mathrm{~g} \cdot \mathrm{Kg}^{-1} \cdot \mathrm{d}^{-1}\right)$, PCMG $\left(0.5 \mathrm{~g} \cdot \mathrm{Kg}^{-1} \cdot \mathrm{d}^{-1}\right)$ and PCLG $\left(0.25 \mathrm{~g} \cdot \mathrm{Kg}^{-1} \cdot \mathrm{d}^{-1}\right)$ were fed with high fat diet and given with different dosage of cholestyramine. The drug was given at 19:00 for once in each day after $2 \mathrm{~h}$ of fasting, continuing for 5 weeks $[14,15]$. The living condition of the rats was observed and their weights and diets were all recorded.

Observation and detection: After the last giving drug, the rats were fasted for $12 \mathrm{~h}$, and then weighted and their vein blood was collected from tail. The serum was obtained after centrifuging at $3,000 \times \mathrm{g}$ for $5 \mathrm{~min}$ at $4^{\circ} \mathrm{C}$. And the $\mathrm{TG}, \mathrm{TC}$, AST and ALT serum levels were detected with detection kits, separately. The rats were sacrificed by cervical vertebra dislocation. Their liver tissues were collected and weighted, and the left lobe of liver was fixed with $10 \%$ formalin, and then treated with the routine embedding with paraffin wax. The tissue was cut into $4 \mu \mathrm{m}$ of sections and then stained with Hematoxylin-Eosin (HE). The right lobe was stored in liquid nitrogen. SOD, MDA, HL and LPL in liver tissue were detected according to liver index $(\%)=($ wet liver weight $(\mathrm{g})) /$ (body weight $(\mathrm{g})) \times 100 \%$.

\section{Statistical analysis}

All data were showed as mean \pm standard deviations $(\bar{x} \pm S)$ and analyzed by SPSS 19.0 software. A value of $\mathrm{P}<0.05$ considered significant difference.

\section{Results}

\section{Pathological change of liver tissue after PC treatment}

Observation: According to Figure 1, the liver tissue was bright red and tough with sharp edge, luster and elasticity in control group (Figure 1A). The liver tissue enlarged obviously in MG (Figure 1B), which showed light yellow with regional yellow-white degeneration, stress capsule, blunt edge and greasiness section. The liver tissue in CG (Figure 1C) was dull red, soft and smooth with sharp edge. Comparing to $\mathrm{MG}$, the conditions of liver tissue were improved with different degrees in PCHG (Figure 1D), PCMG (Figure 1E) and PCLG (Figure $1 \mathrm{~F})$, showing as dosage-dependence.

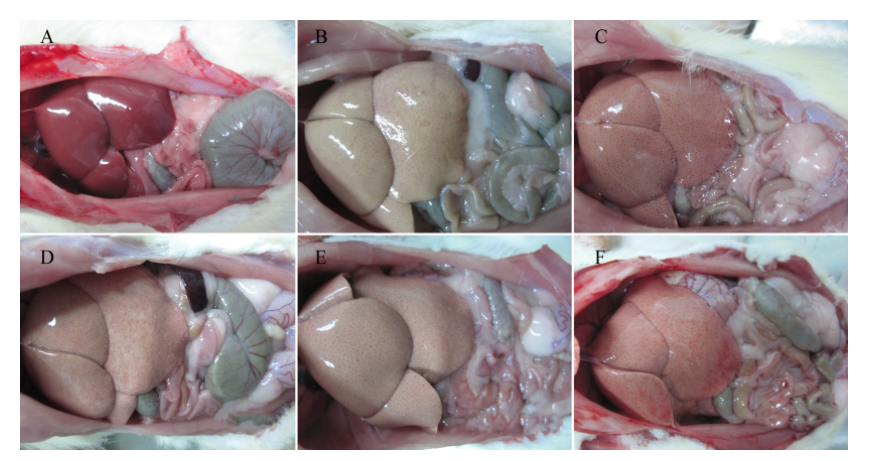

Figure 1. The appearance of liver changed in each group. A) Control group; B) Model group; C) Cholestyramine group; D) Pleurotus eryngii cellulose low-dose group; E) Pleurotus eryngii cellulose middle-dose group; F) Pleurotus eryngii cellulose high-dose group.

Histopathological observation: The structure of liver tissue was normal in control group (Figure 2A). It showed that the hepatocytes arranged radically around the central vein without fatty degeneration, clear hepatic sinusoid, tidy hepatic cord and no fat vacuole in the liver tissue. The lobe of liver tissue in $\mathrm{MG}$ 
(Figure 2B) showed unclear edge, swelling and round hepatocytes, no hepatic sinusoid, disorganized hepatic cord and some fat vacuoles in the liver tissue with serious fatty degeneration. The structure of liver tissue in CG (Figure 2C) was normal, they showed hepatocytes arranged radically around the central vein without fatty degeneration, slightly swelling hepatocytes and no fat vacuole in the liver tissue. The lob of live tissue in PCLG (Figure 2D) showed unclear edge, disorganized hepatic cord and no fat vacuoles in the liver tissue, comparing to PCLG, the conditions were improved in PCMG (Figure 2E), the hepatocytes were without fatty degeneration, and there was no fat vacuole in liver tissue. Comparing to PCLG and PCMG, the liver tissue in PCHG (Figure 2F) was similar to that in control group but with few neutrophils.

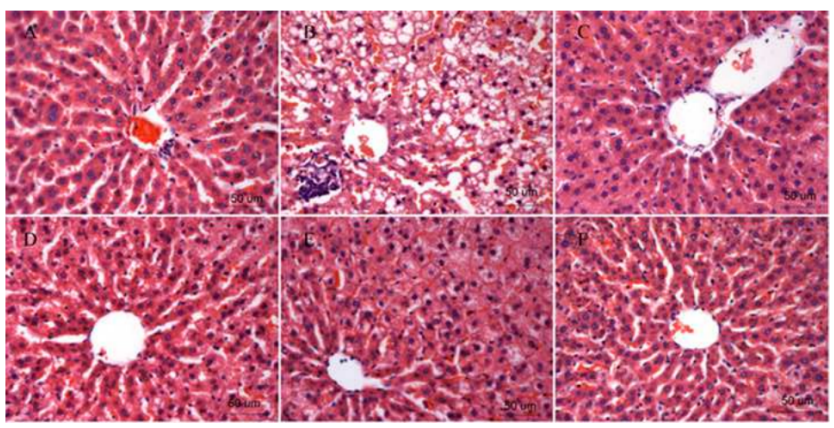

Figure 2. The histopathology of liver changed in each group (HE, 400X). A) Control group; B) Model group; C) Cholestyramine group; D) Pleurotus eryngii cellulose low-dose group; E) Pleurotus eryngii cellulose middle-dose group; F) Pleurotus eryngii cellulose high-dose group.

Changes of serum lipid and liver function after PC treatment: Comparing to control group, serum ALT, AST, TC and TG increased significantly in $\mathrm{MG}(\mathrm{P}<0.01)$ (Table 1), which indicated fatty liver model was established successfully [16]. After intervention for 5 weeks, comparing to $\mathrm{MG}$, serum lipid decreased significantly $(\mathrm{P}<0.01)$ in rats treated with $\mathrm{PC}$
(Table 1), which showed as dosage-dependence. Serum TC in MG was $2.56 \pm 0.16 \mathrm{mmol} / \mathrm{L}$, while it was $1.96 \pm 0.066$ $\mathrm{mmol} / \mathrm{L}, 2.06 \pm 0.08 \mathrm{mmol} / \mathrm{L}$ and $2.19 \pm 0.09 \mathrm{mmol} / \mathrm{L}$ in in PCHG, PCMG and PCLG separately. Serum levels of ALT in MG was $92.23 \pm 10.6 \mathrm{U} / \mathrm{L}$, while it was $49.01 \pm 4.17 \mathrm{U} / \mathrm{L}$, $59.33 \pm 6.23 \mathrm{U} / \mathrm{L}$ and $72.23 \pm 5.99 \mathrm{U} / \mathrm{L}$ in PCHG, PCMG and PCLG separately. Comparing to $\mathrm{CG}$, there was no significant difference in serum lipid or transaminase of PCHG, which indicated that $\mathrm{PC}$ had preventive effects on fatty liver.

Liver index and relative enzyme activity after PC treatment: Comparing to $\mathrm{MG}, \mathrm{PC}$ could improve after activity of SOD plus and HL, decrease the MDA content and did not have any effect on the activity of LPL, the deposition of lipid in liver tissue and the liver index induced by lipid metabolism disorder, significantly $(\mathrm{P}<0.01)$ (Table 2$)$. The effects showed as dosage-dependence.

\section{Discussion}

Liver was the main location of lipid metabolism. With high fat diet, the exogenous fat increased and the hepatocyte esterified, this led to the increasing of TG, decreasing of TG degradation and increase of TG flowing into liver. Excessive intake of cholesterol and free fatty acids would induce the damage of cytotoxicity and hepatocellular function and lead to the decrease of hepatocellular transport capacity of TG, which showed hepatic dysfunction and serum levels of TC and TG increasing abnormally $[17,18]$. Cholestyramine, as the preventive and positive control drug, showed multiple functions such as exchanging and absorbing the intestinal bile salts, blocking the enterohepatic circulation of cholic acid, accelerating the decomposing of cholesterol into bile salt and decreasing the TC levels. Additionally, the intestinal absorption of cholesterol needed the emulsification of bile salt. After the adsorption and excretion of bile salts, the readsorption of cholesterol was inhibited, causing decreasing of the TC.

Table 1. The effect of PC on blood lipid and liver function in rat.

\begin{tabular}{|c|c|c|c|c|c|}
\hline Group & Number & TC (mmol/L) & TG (mmol/L) & ALT (U/L) & AST (U/L) \\
\hline CT & 8 & $1.81 \pm 0.12$ & $0.56 \pm 0.07$ & $39.30 \pm 2.36$ & $117.10 \pm 8.47$ \\
\hline MG & 8 & $2.56 \pm 0.16^{b}$ & $1.51 \pm 0.06^{b}$ & $92.23 \pm 10.6^{b}$ & $208.36 \pm 19.33^{b}$ \\
\hline CG & 8 & $1.89 \pm 0.09^{d}$ & $0.86 \pm 0.09^{b d}$ & $44.08 \pm 2.61^{\mathrm{bd}}$ & $139.03 \pm 6.06^{\mathrm{bd}}$ \\
\hline PCHG & 8 & $1.96 \pm 0.06^{b d}$ & $1.00 \pm 0.09^{\mathrm{bde}}$ & $49.01 \pm 4.17^{\text {bde }}$ & $143.46 \pm 9.16^{\mathrm{bd}}$ \\
\hline PCMG & 8 & $2.06 \pm 0.08^{\mathrm{bdf}}$ & $1.12 \pm 0.11^{\mathrm{bdf}}$ & $59.33 \pm 6.23^{\text {bdf }}$ & $157.78 \pm 10.96^{\mathrm{bdf}}$ \\
\hline PCLG & 8 & $2.19 \pm 0.09^{b d f}$ & $1.28 \pm 0.05^{\mathrm{bdf}}$ & $72.23 \pm 5.99^{\mathrm{bdf}}$ & $180.00 \pm 7.54^{\mathrm{bdf}}$ \\
\hline
\end{tabular}

Note: aP<0.05, ${ }^{b} P<0.01$ vs. control group; ${ }^{\mathrm{C}} \mathrm{P}<0.05$, ${ }^{\mathrm{d}} \mathrm{P}<0.01$ vs. model group; ${ }^{\mathrm{e}} \mathrm{P}<0.05$, ${ }^{\mathrm{P}}<0.01$ vs. cholestyramine group.

Table 2. The effect of $P C$ on liver index, SOD, MDA in rats' liver $(\bar{x} \pm s)$.

\begin{tabular}{|c|c|c|c|c|c|c|}
\hline Group & Number & SOD (U/mgprot) & MDA (nmol/mgprot) & HL (U/mgprot) & LPL (U/mgprot) & Liver index(\%) \\
\hline
\end{tabular}




\begin{tabular}{lllllll}
\hline TG & 8 & $21.70 \pm 1.51$ & $14.35 \pm 1.48$ & $2.62 \pm 0.18$ & $1.46 \pm 0.25$ & $3.09 \pm 0.38$ \\
\hline MG & $8^{\mathrm{b}}$ & $9.29 \pm 1.79^{\mathrm{b}}$ & $40.14 \pm 3.07^{\mathrm{b}}$ & $1.63 \pm 0.14^{\mathrm{b}}$ & $0.85 \pm 0.05^{\mathrm{b}}$ & $4.56 \pm 0.61^{\mathrm{b}}$ \\
\hline CG & 8 & $18.23 \pm 2.60^{\mathrm{bd}}$ & $16.69 \pm 1.26^{\mathrm{ad}}$ & $2.06 \pm 0.25^{\mathrm{d}}$ & $1.43 \pm 0.33^{\mathrm{d}}$ & $3.94 \pm 0.27^{\mathrm{ac}}$ \\
\hline PCHG & 6 & $15.87 \pm 1.50^{\mathrm{bd}}$ & $35.36 \pm 4.52^{\mathrm{bcf}}$ & $1.98 \pm 0.21^{\mathrm{bd}}$ & $0.86 \pm 0.09^{\mathrm{a}}$ & $3.64 \pm 0.31^{\mathrm{ad}}$ \\
\hline PCMG & 7 & $14.10 \pm 2.83^{\mathrm{bde}}$ & $41.52 \pm 6.36^{\mathrm{bf}}$ & $1.79 \pm 0.10^{\mathrm{bce}}$ & $0.71 \pm 0.05^{\mathrm{bcf}}$ & $4.02 \pm 0.29^{\mathrm{bc}}$ \\
\hline PCLG & 6 & $13.45 \pm 1.89^{\mathrm{bdf}}$ & $44.21 \pm 1.88^{\mathrm{bcf}}$ & $1.60 \pm 0.37^{\mathrm{be}}$ & $0.46 \pm 0.06^{\mathrm{bf}}$ & $4.23 \pm 0.39^{\mathrm{b}}$ \\
\hline
\end{tabular}

Note: ${ }^{\mathrm{a} P}<0.05,{ }^{\mathrm{b}} \mathrm{P}<0.01$ vs. control group; ${ }^{\mathrm{C}}<0.05$, ${ }^{\mathrm{d}} \mathrm{P}<0.01$ vs. model group; ${ }^{\mathrm{e}} \mathrm{P}<0.05,{ }^{\mathrm{f}} \mathrm{P}<0.01$ vs. cholestyramine group.

In our study, after 5 weeks treated with PC, the serum TC and TG in the rats decreased significantly, and their ALT and AST recovered obviously. The preventive effect in $\mathrm{PCHG}$ was obvious, the same as the $\mathrm{CG}$, which indicated that $\mathrm{PC}$ could reduce the absorption of exogenous fat and TC and TG serum levels to release the metabolic pressure of liver for fat. This result was consistent with the report of Wang et al., whose study showed that the dietary fiber could improve intestinal peristalsis, shorten the residence time of food in intestine, and reduce the absorption and metabolism of fat, and decrease TC and TG levels in liver [19].

It was still unclear about the pathogenesis of fat liver that was usually induced by the increase of synthesis and decrease of oxidation of hepatocellular fat. In our study, MDA in the liver tissue increased in $\mathrm{MG}$ group comparing to control group, while SOD activity decreased significantly $(\mathrm{P}<0.01)$, which indicated that the hepatocellular oxygen consumption increased and lots of free radicals increased leading to lipid peroxidation enhance and increasing of MDA. After treating with PC, SOD activity was improved in PCMG and $\mathrm{PCHG}$, while MDA was inhibited significantly $(\mathrm{P}<0.05)$. This was in accordance with previous study, showing that PC was with the ability to clear the free radicals, improve the antioxidant capacity, regulate and improve the balance of metabolism of the free radicals [20].

In conclusion, PC had the abilities of improving antioxidant capacity of liver tissue, reducing the deposition of fat and preventing hyperlipidemia and fatty liver.

\section{Acknowledgement}

The Natural Science Fund Project of Zhangzhou City (No. ZZ2017J19). The Natural Science Fund Project of Fujian Province (No. 2016J05083). The Education Scientific Research Project of Young Teachers in Fujian Province (JA15306).

\section{References}

1. Lu RJ, Ren D X , Yan M. Progress in studies of the pathogenesis and therapy of the fatty liver J Gansu Sci 2001; 13: 57-64.

2. Chang XM, Yun JW. Advances in the prevention and treatment of fatty liver disease. Shijie Huaren Xiaohua Zazhi 2009; 17: 3573-3578.

3. Diao ZM, Wei KJ, Wu BF. Mushroomology. Qinghai People Press, Xining, China 2006; 114-115.
4. Lee CY, Park JE, Kim BB, Kim SM, Ro HS. Determination of mineral components in the cultivation substrates of edible mushrooms and their uptake into fruiting bodies. Mycobiology 2009; 37: 109-113.

5. Mizutani $\mathrm{T}$, Inatomi $\mathrm{S}$, Inazu $\mathrm{A}$, Kawahara $\mathrm{E}$. Hypolipidemic effect of Pleurotus eryngii extract in fatloaded mice. J Nutr Sci Vitaminol 2010; 56: 48-53.

6. Mori K, Kobayashi C, Tomita $\mathrm{T}$, Inatomi S, Ikeda M. Antiatherosclerotic effect of the edible mushrooms Pleurotus eryngii (Eringi), Grifola frondosa (Maitake), and Hypsizygus marmoreus (Bunashimeji) in apolipoprotein E-deficient mice. Nutr Res 2008; 28: 335-342.

7. Ike K, Kameyama N, Ito A, Imai S. Induction of a THelper 1 (Th1) immune response in mice by an extract from the Pleurotus eryngii (Eringi) mushroom. J Med Food 2012; 15: 1124-1128.

8. Choi JH, Kim HG, Jin SW, Han EH, Khanal T, Do MT, Hwang YP, Choi JM, Chun SS, Chung YC, Jeong TC, Jeong HG. Topical application of Pleurotus eryngii extracts inhibits 2, 4-dinitrochlorobenzene-induced atopic dermatitis in NC/Nga mice by the regulation of Th1/Th2 balance. Food Chem Toxicol 2013; 53: 38-45.

9. Shang $X$, Tan Q, Liu R, Yu K, Li P ,Zhao GP. In vitro anti-Helicobacter pylori effects of medicinal mushroom extracts, with special emphasis on the Lion's Mane mushroom, Hericium erinaceus (higher Basidiomycetes). Int J Med Mushrooms 2013; 15: 165-174.

10. Li SQ, Shah NP. Antioxidant and antibacterial activities of sulphated polysaccharides from Pleurotus eryngii and Streptococcus thermophilus ASCC 1275. Food Chem 2014; 165: 262-270.

11. Krupodorova T, Rybalko S, Barshteyn V. Antiviral activity of Basidiomycete mycelia against influenza type A (serotype H1N1) and herpes simplex virus type 2 in cell culture. Virologica Sinica 2014; 29: 284-290.

12. Li JW, Yang YX. Review in defining dietary fiber and analysis methods. Food Sci 2007; 28: 350-354.

13. Wang AN, Zhu HL, Wu LG, Wei SX. The function modification and application of dietary fiber. J Henan Univ Technol 2009; 30: 89-93.

14. Wand H, Yang LY, Cao XD, Qian LN, Wu SQ, Wang $\mathrm{XS}$.Establishment of the SD rat models of hyperlipemia 
and fatty liver. Heilongjiang Animal Sci Veterinary Med 2015; 3: 172-174.

15. Zhong L, Fan JG, Wang GL, Wu WQ, Shi Q. The establishment of non-alcohlic steatohepatitis (NASH) animal model. Laboratory Animal Science and Administration 2000; 17: 16-20.

16. Jia LC, Ma LX, Wu LP, Wang Y, Li ZQ, Yang HR. The experimental study on the curative effects of balance diets on the fatty liver. Modern Preventive Med 2006; 33: 2254-2258.

17. Yi GY, Yang TS, Li YZ, Zhang HW. The hepatic effects of combination therapy of probucol and atorvastatin in spontaneously hypertensive rats with high lipids diet. Chin J Arteriosclerosis 2009; 17: 117-121.

18. Dong $\mathrm{CF}, \mathrm{Wu} \mathrm{GH}$. The analysis of change in blood fat, liver function index for the patients with fatty liver. Laboratory Med 2005; 20: 160-161.
19. Wang WH, Yang JJ, Wang J. The effects of low fat high diet fiber on lipids and anti-oxidation in mice liver. Modern Preventive Med 2010; 37: 2644-2646.

20. Ye J, Xiao M T, Liu Q, Tang XC, Xiao B. Effect of Gracilaria dietary fiber on lipid peroxides activity in senile mice. J Huaqiao Univ 2011; 32: 58-61.

\section{*Correspondence to}

Yu-Tian Pan

Engineering Technological Center of Mushroom Industry

Minnan Normal University

Zhangzhou

Fujian

PR China 